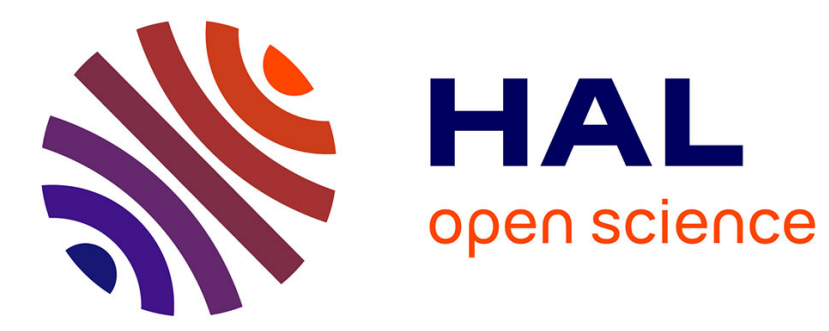

\title{
Teaching Bayesian Behaviours to Video Game Characters
}

Ronan Le Hy, Anthony Arrigoni, Pierre Bessiere, Olivier Lebeltel

\section{To cite this version:}

Ronan Le Hy, Anthony Arrigoni, Pierre Bessiere, Olivier Lebeltel. Teaching Bayesian Behaviours to Video Game Characters. Robotics and Autonomous Systems, 2004, 47, 47, pp.177-185. inria00182073

\section{HAL Id: inria-00182073 https://hal.inria.fr/inria-00182073}

Submitted on 30 Oct 2007

HAL is a multi-disciplinary open access archive for the deposit and dissemination of scientific research documents, whether they are published or not. The documents may come from teaching and research institutions in France or abroad, or from public or private research centers.
L'archive ouverte pluridisciplinaire HAL, est destinée au dépôt et à la diffusion de documents scientifiques de niveau recherche, publiés ou non, émanant des établissements d'enseignement et de recherche français ou étrangers, des laboratoires publics ou privés. 


\title{
Teaching Bayesian Behaviours to Video Game Characters
}

\author{
Ronan Le Hy ${ }^{a}$ Anthony Arrigoni ${ }^{a}$ Pierre Bessière ${ }^{a}$ Olivier Lebeltel ${ }^{\text {a }}$ \\ a $G R A V I R / I M A G$ \\ INRIA Rhône-Alpes, ZIRST \\ 38330 Montbonnot, France
}

\begin{abstract}
This article explores an application of Bayesian Programming to behaviours for synthetic video games characters. We address the problem of real-time reactive selection of elementary behaviours for an agent playing a first person shooter game. We show how Bayesian Programming can lead to condensed and easier formalisation of finite state machine-like behaviour selection, and lend itself to learning by imitation, in a fully transparent way for the player.
\end{abstract}

Key words: bayesian programming, automaton, synthetic character PACS:

\section{Introduction}

Today's video games feature synthetic characters involved in complex interactions with human players. As John Laird sums it up [1], a synthetic character may have one of many different roles: tactical enemy, partner for the human, strategic opponent, simple unit amongst many, commenter... In all of these cases, the game developer's ultimate objective is for the synthetic character to act like a human player.

We are interested in a particular type of synthetic character, which we call a bot in the rest of this article. It is a player for a first person shooter game named Unreal Tournament augmented with the Gamebots control framework [2] (see figure 1). This framework provides a tridimensional environment in which players have to fight each other, taking advantage of resources such as weapons and health bonuses available in the arena. We believe, with Laird [1,3], that this kind of computer game provides a challenging ground for the development of human-level AI.

Email address: lehy@imag.fr (Ronan Le Hy).
After listing our practical objectives, we will present our bayesian model. We will show how we use it to specify by hand a behaviour, and how we use it to learn a behaviour. We will tackle learning by example using a high-level interface, and then the natural controls of the game. We will show that it is possible to map the player's actions onto bot states, and use this reconstruction to learn our model. Finally, we will come back to our objectives as a conclusion.

\subsection{Objectives}

Our core objective is to propose an efficient way to specify a behaviour for our bot. This can be broken down into several criteria that hold either for the developers or for the player.

\subsubsection{Development Team's Viewpoint}

Programming efficiency. One crucial concern for the programmer is productivity: he needs both expressivity and simplicity of the behaviour programming system.

Limited computation requirements. The processing time alloted to $\mathrm{AI}$ in games is typ- 


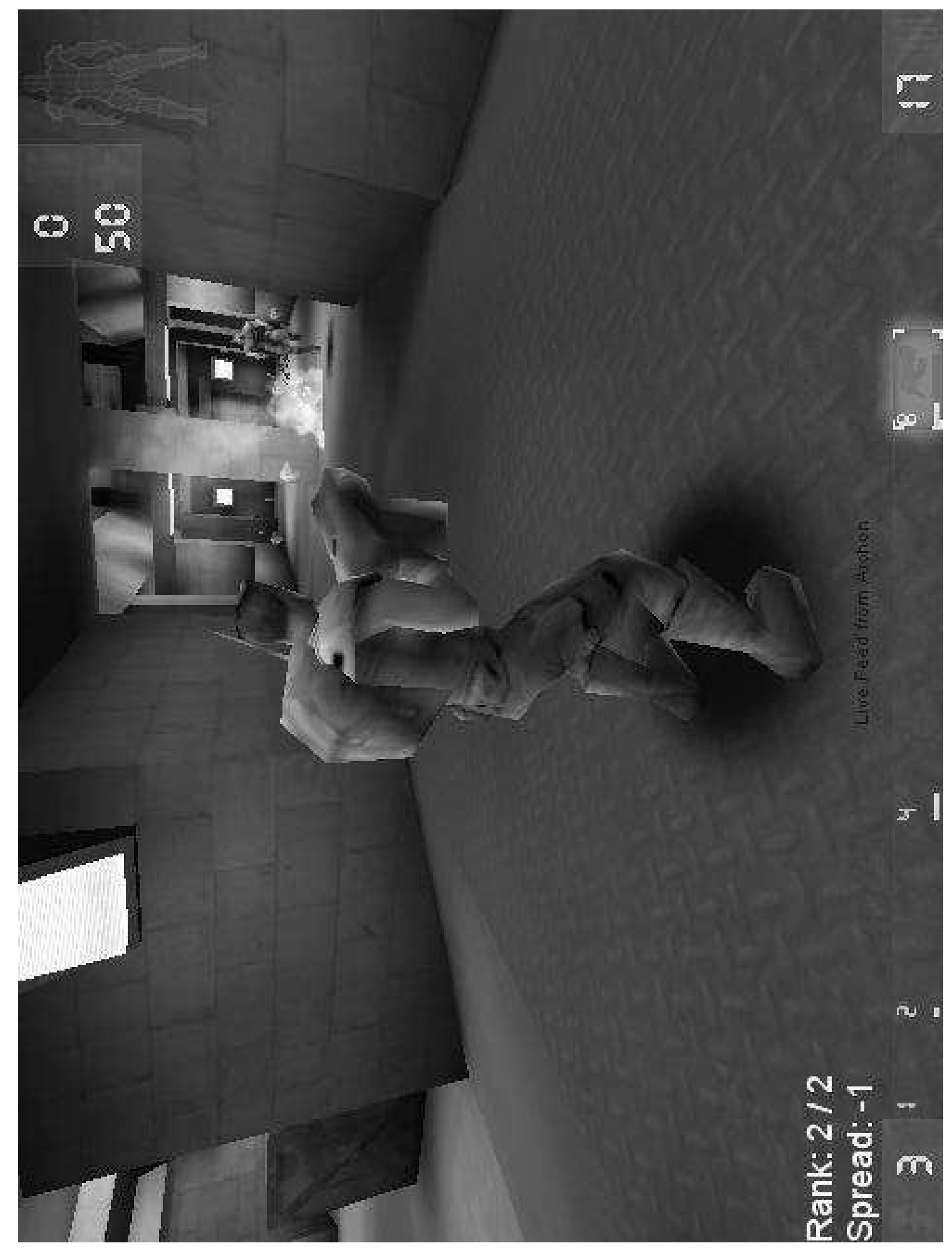

Fig. 1. Unreal Tournament and the Gamebots environment. 
ically between $10 \%$ and $20 \%$ of the total processing time [4]; therefore it is important for the behaviour system to be light in terms of computation time.

Design / development separation. The industrial development scheme often draws a separation between game designers and software developers. The system should allow the designers to describe behaviours at a high conceptual level, without any knowledge of the engine's internals.

Behaviour tunability. The ability to program a variety of different behaviours, and to adjust each of them without having to modify the system's back end is essential to the designer.

\subsubsection{Player's Viewpoint}

"Humanness". As defined by Laird [5], this implies the illusion of spatial reasoning, memory, common sense reasoning, using goals, tactics, planning, communication and coordination, adaptation, unpredictability... One important criterion for the player is that the synthetic character does not cheat; its perceptions and actions should be as much as possible like a human player's.

Behaviour learning. This feature is gradually finding its place in modern games: the player can adjust its synthetic partners' behaviour. The behaviour system should therefore support learning.

\subsection{Technical Framework}

As mentioned earlier, we used the Gamebots framework to conduct our experiments. This implies that our bot communicates with Unreal Tournament via a text protocol on a Unix socket. It receives messages covering its perceptions: its position and speed, health level, ammunition, visible opponents and objects, etc. In return, it sends actions: move to a given point, rotate, change weapon...

The environment is perceived by the bot as a graph, of which nodes are characteristic points of the topology and various objects. The bot perceives only what is in its field of vision.

As our objectives and framework have been exposed, we shall now proceed to explicit our model of behaviour selection, and discuss its interest for the specification and learning of behaviours.

\section{Bayesian Model}

Before examining our particular bot model, we review in the next section the principles of Bayesian Programming [6].

\subsection{Bayesian Programming}

Rational reasoning with incomplete and uncertain information is quite a challenge. Bayesian Programming addresses this challenge, and relies upon a well established formal theory: the probability theory [7]. As a modelling tool, it encompasses the framework of Bayesian Networks [8].

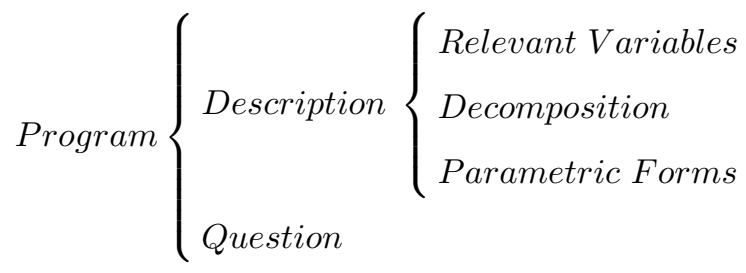

Fig. 2. structure of a Bayesian Program

In our framework, a Bayesian Program is made of two parts: a description and a question.

The description can be viewed as a knowledge base containing the a priori information available about the problem at hand. It is essentially a joint probability distribution. The description is made up of three components: 1) A set of relevant variables on which the joint distribution is defined. Typically, variables are motor, sensory or internal. 2) A decomposition of the joint distribution as a product of simpler terms. It is obtained by applying Bayes theorem and taking advantage of the conditional independencies that may exist between variables. 3) The parametric forms assigned to each of the terms appearing in the decomposition (they are required to compute the joint distribution).

Given a distribution, it is possible to ask questions. Questions are obtained first by partitioning the set of variables into three sets: (1) Searched: the searched variables, (2) Known: the known variables, and (3) Free: the free variables. A question is then defined as the distribution:

$P($ Searched $\mid$ Known $)$

Given the description, it is always possible to answer a question, i.e. to compute the probability 
distribution $P($ Searched $\mid$ Known $)$. To do so, the following general inference rule is used:

$$
\begin{aligned}
& P(\text { Searched } \mid \text { Known }) \\
& =\frac{\sum_{\text {Free }} P(\text { Searched Free Known })}{P(\text { Known })} \\
& =\frac{1}{Z} \times \sum_{\text {Free }} P(\text { Searched Free Known })
\end{aligned}
$$

where $Z$ is a normalisation term. As such, the inference is computationally expensive (Bayesian inference in general has been shown to be NP-Hard [9]). A symbolic simplification phase can reduce drastically the number of sums necessary to compute a given distribution. However the decomposition of the preliminary knowledge, which expresses the conditional independencies of variables, still plays a crucial role in keeping the computation tractable.

\subsection{Modelling our Bot}

\subsubsection{Bayesian Program}

Our particular bot behaviour uses the following bayesian program.

\subsubsection{Relevant Variables}

$S_{t}$ : the bot's state at time $t$. One of Attack, SearchWeapon, SearchHealth, Explore, Flee, DetectDanger. These states correspond to elementary behaviours, in our example programmed in a classic procedural fashion.

$S_{t+1}$ : the bot's state at time $t+1$.

$H$ : the bot's health level at $t$.

$W$ : the bot's weapon at $t$.

$O W$ : the opponent's weapon at $t$.

$H N$ : indicates whether a noise has been heard recently at $t$.

$N E$ : the number of close enemies at $t$.

$P W$ : indicates whether a weapon is close at $t$.

$P H$ : indicate whether a health pack is close at $t$. The elementary motor commands of the bot are the values of variables $S_{t+1}$ and $S_{t}$. They include an attack behaviour, in which the bot shoots at an opponent while maintaining a constant distance to him and strafing; a fleeing behaviour, which consists in trying to escape (locally) an opponent; behaviours to fetch a weapon or a health bonus the bot noticed in its environment; and a behaviour to detect possible opponents outside the current field of view of the bot; and behaviour to navigate around the environment and discover unexplored parts of it.

2.2.1.2 Decomposition The joint distribution is decomposed as:

$$
\begin{aligned}
& P\left(S_{t} S_{t+1} H W O W H N N E P W P H\right) \\
&=P\left(S_{t}\right) \\
& P\left(S_{t+1} \mid S_{t}\right) \\
& P\left(H \mid S_{t+1}\right) \\
& P\left(W \mid S_{t+1}\right) \\
& P\left(O W \mid S_{t+1}\right) \\
& P\left(H N \mid S_{t+1}\right) \\
& P\left(N E \mid S_{t+1}\right) \\
& P\left(P W \mid S_{t+1}\right) \\
& P\left(P H \mid S_{t+1}\right)
\end{aligned}
$$

To write the above, we make the hypothesis that knowing $S_{t+1}$, any sensory variable is independent to each other sensory variable (which makes our model a particular sort of Hidden Markov Model). Although it may seem to reduce the expressivity of our model, it allows to specify it in a very condensed way; this point will be emphasised upon in section 2.2.2.

\subsubsection{Parametric Forms}

- $P\left(S_{t}\right)$ : unknown (unspecified)

- $P\left(S_{t+1} \mid S_{t}\right)$ : table (this table will be defined in section 2.2.2)

- $P\left(\right.$ Sensor $\left.\mid S_{t+1}\right)$ with Sensor each of the sensory variables: tables

2.2.1.4 Identification Identification of the parametric forms is done either by manually writing the tables, or by learning them. We describe these two processes in sections 3 (Specifying a Behaviour) and 4 (Learning a Behaviour).

2.2.1.5 Question Every time our bot has to take a decision, the question we ask to our model is:

\section{$P\left(S_{t+1} \mid S_{t} H\right.$ W OW HN NE PW PH $)$}

Knowing the current state and the values of the sensors, we want to know the new state the bot 
should switch into. This question leads a probability distribution, on which we draw a value to decide the actual new state. This state translates directly into an elementary behaviour which is applied to the bot.

\subsubsection{Inverse Programming}

We shall now emphasise the peculiarities of our method to specify behaviours, compared to one using simple finite state machines (FSMs). The problem we address is, knowing the current state and the sensors' values, to determine the next state: this is actually naturally accomplished using an FSM.

Let us consider the case where each of our $n$ sensory variables has $m_{i}(1 \leq i \leq n)$ possible values. In an FSM modelling a behaviour [10,11], we would have to specify, for each state, a transition to each state, in the form of a logical condition on the sensory variables.

It means that the programmer has to discriminate amongst the $\prod_{i} m_{i}$ possible sensory combinations to describe the state transitions. Not only does this pose the difficult problem of determining the appropriate transitions, but it raises the question of convenient formalised representation. This approach could actually lead to several implementations, but will possibly [12] result in a script resembling the following:

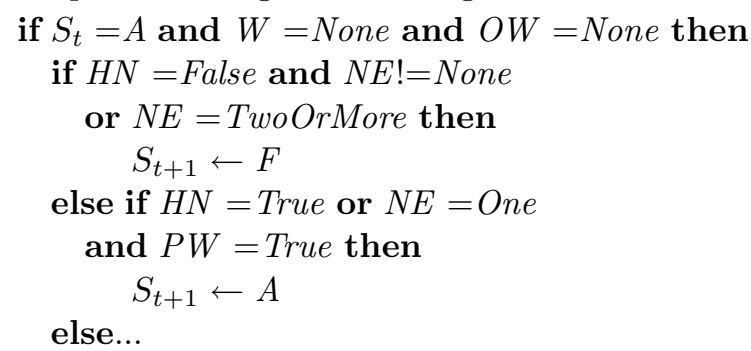

This kind of script is hard to write and hard to maintain.

In contrast, our approach consists in giving, for each sensory variable, for each possible state, a distribution (i.e. $m_{i}$ numbers summing to 1 ). In practice, we write tables like table 1 , which represents $P\left(H \mid S_{t+1}\right)$. Values of $H$ are enumerated in the first column, those of $S_{t+1}$ in the first line; cells marked $x$ are computed so that each column sums to 1 .

Moreover, instead of specifying the conditions that make the bot switch from one state to another, we specify the (probability distribution of the) sensors' values when the bot goes into a given state. This way of specifying a sensor under the hypothesis that we know the state is what makes us call our method "inverse programming".

Although somewhat confusing at first, this is the core advantage of our way to specify a behaviour. As a matter of fact, we have to describe separately the influence of each sensor on the bot's state, thereby reducing drastically the quantity of needed information. Furthermore, it becomes very easy to incorporate a new sensory variable into our model: it just requires to write an additional table, without modifying the existing ones. Finally, the number of figures we need in order to specify a behaviour is $s^{2}+s n m$, where $s$ is the number of states, $n$ the number of sensory variables, and $m$ the average number of possible values for the sensory variables. It is therefore linear in the number of variables (assuming $m$ constant).

\begin{tabular}{|c|r||r||r||r||r||r|}
\hline$H \backslash S_{t+1}$ & $A$ & $S W$ & $S H$ & $E x$ & $F$ & $D D$ \\
\hline Low & 0.001 & 0.1 & $x_{3}$ & 0.1 & 0.7 & 0.1 \\
\hline Medium & 0.1 & $x_{2}$ & 0.01 & $x_{4}$ & 0.2 & $x_{5}$ \\
\hline High & $x_{1}$ & $x_{2}$ & 0.001 & $x_{4}$ & 0.1 & $x_{5}$ \\
\hline
\end{tabular}

$P\left(H \mid S_{t+1}\right)$

\section{$3 \quad$ Specifying a Behaviour}

\subsection{Basic specification}

A behaviour can be specified by writing the tables corresponding to $P\left(S_{t+1} \mid S_{t}\right)$ and $P\left(\right.$ Sensor $\left.\mid S_{t+1}\right)$ (for each sensory variable). Let us consider for instance table 1, which gives the probability distribution for $H$ knowing $S_{t+1}$. We read the first column this way: given the fact that the bot is going to be in state Attack, we know that it has a very low probability (0.001) to have a low health level, a medium probability (0.1) to have a medium health level, and a strong chance $(x=1-0.001-$ 0.1 ) to have a high health level.

This form of specification allows us to formalise conveniently the constraints we want to impose on the behaviour, in a condensed format, and separately on each sensory variable. For instance, table 1 formalises the relation of the bot's health level to its state: if it starts attacking, then its health is rather high; if it starts searching for a health pack, then its health is very probably 
low; if it starts fleeing, then its health is probably rather low, but with a high degree of uncertainty. All tables on the sensory variables are built on the same pattern; the one giving $P\left(S_{t+1} \mid S_{t}\right)$ (see table 2 ) is special. It gives some sort of basic transition table; i.e. it answers in a probabilistic way the question: knowing nothing but the current state, what will be the next state?

\subsection{Results}

Several observations can be made when out bots are playing the game. The first is that their behaviour corresponds to what we want: the behaviour switching occurs reasonably, given the evolution of the sensory variables. The second is that they can't compete with humans playing the game. Noting this allows to pinpoint the fact that our method's interest mostly resides in the gain

\begin{tabular}{|c|c|c|c|c|c|c|}
\hline$S_{t+1} \backslash S_{t}$ & $A$ & $S W$ & $S H$ & $E x$ & $F$ & $\begin{array}{l}\text { our method's interest mostly resides in the gal } \\
D D \text { of ease and power in the desion of behaviours. }\end{array}$ \\
\hline$A$ & $x_{1}$ & $x_{2}$ & $x_{3}$ & $x_{4}$ & $x_{5}$ & $x_{6 \text { does not pretend to overcome the limitations of }}$ \\
\hline$S W$ & $10^{-5}$ & $x_{2}$ & $10^{-5}$ & $10^{-5}$ & $10^{-5}$ & 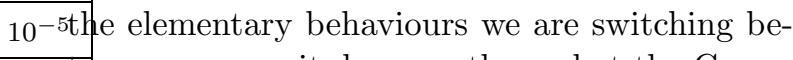 \\
\hline$S H$ & $10^{-5}$ & $10^{-5}$ & $x_{3}$ & $10^{-5}$ & $10^{-5}$ & $10^{-5}$ tween, nor can it do more than what the Gam \\
\hline$E x$ & $10^{-5}$ & $10^{-5}$ & $10^{-5}$ & $x_{4}$ & $10^{-5}$ & $10^{-5}$ action. Therefore, what we aimed for, and finally \\
\hline$F$ & $10^{-5}$ & $10^{-5}$ & $10^{-5}$ & $10^{-5}$ & $x_{5}$ & $10^{-5}$ obtained, is a reliable, practical and efficient way \\
\hline$D D$ & $10^{-5}$ & $10^{-5}$ & $10^{-5}$ & $10^{-5}$ & $10^{-5}$ & $\begin{array}{l}x_{6}^{\text {to }} \text { specify the real-time selection of elementar } \\
\text { behaviours. }\end{array}$ \\
\hline
\end{tabular}

$P\left(S_{t+1} \mid S_{t}\right)$

The answer our sample table gives is: tend to stay in your current state (notice the $x$ s on the diagonal) or switch to attack (notice the $x$ s on the first line) with the same high probability; switch to other states with a very low probability $\left(10^{-5}\right.$ - which in our example we found to be representative of "very low").

Again, this makes a parallel with an FSM with probabilistic transitions: with our transition table $P\left(S_{t+1} \mid S_{t}\right)$, we give a basic automaton upon which we build our behaviour by fusing the tendencies given separately on each sensory variable.

\subsection{Tuning the behaviour}

Tuning our behaviour amounts to tuning our probability distributions. For instance, to create a berserk character that is insensible to its health level, we put only uniform distributions (i.e. in our notation, only $x \mathrm{~s})$ in table $P\left(H \mid S_{t+1}\right)$. A berserk is also very aggressive, so the transition table we proposed in table 2 is quite adapted. A transition table for a more prudent character would not have those $x$ s on the first line, so that the state $A$ would not be particular.

To create a unique behaviour, we therefore have to review all our tables, i.e. the influence of each sensory variable on the character according to the said behaviour.
Our attempt to tune the behaviour shows that the differences between our 'reasonable' bot and our 'aggressive' bot are visible, and correspond to what we tried to specify in the tables. For instance, the aggressive bot is excited by the presence of several opponents, whereas this situation repels the reasonable bot; and the aggressive bot is not discouraged to attack when its health level goes low.

\section{Learning a Behaviour}

Our goal now is to teach the bot a behaviour, instead of specifying all the probability distributions by hand. It requires to be able to measure at each instant sensory and motor variables of the controlled bot. In particular, it is necessary to determine the state $S_{t}$ at each instant. It can be done by letting the player specify it directly in real time, or by inferring it from his natural actions in the game.

\subsection{Selecting behaviours}

This form of learning by example presents a simple interface to the player, shown on figure 3.

The player controls in real time the elementary behaviour that the bot executes, by using buttons that allow switching to any state with a mouse click. In addition to the ordinary Unreal Tournament window on the right, part of the internal 


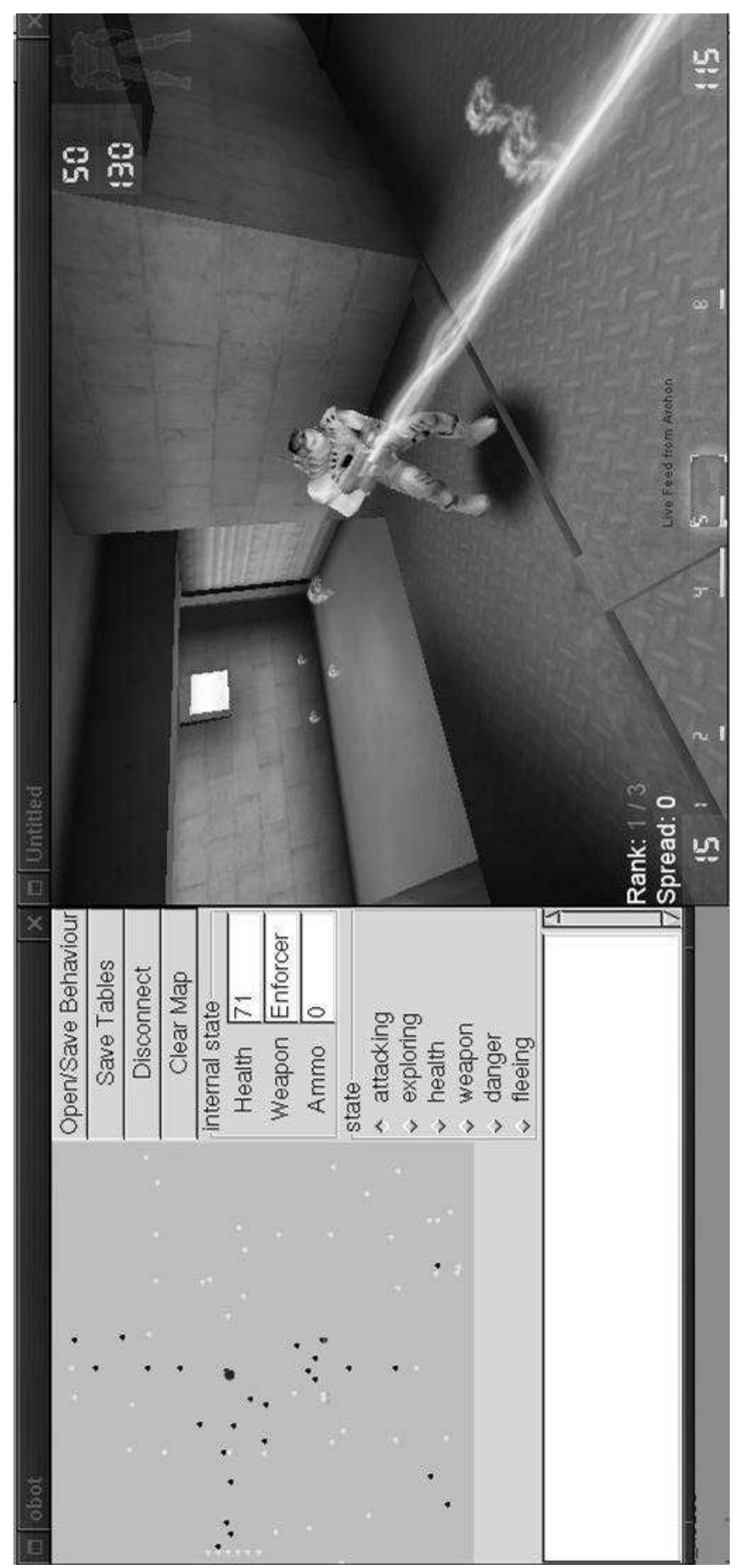

Fig. 3. Interface used to teach the bot: on the right is the normal Unreal Tournament window showing our bot; on the left is our interface to control the bot. 
state of the bot is summed up in the learning interface on the left.

\subsection{Recognising behaviours}

While the previous method of teaching a behaviour works, it deprives the player of the interface he is used to; his perceptions and motor capabilities are mostly adjusted to the bot's. In order to solve this problem, it is possible to give the player the natural interface of the game, and try to recognise in real time the behaviour he is following.

To recognise the human's behaviour from his lowlevel actions, we use a heuristic programmed in a classical imperative fashion. It involves identifying each behaviour's critical variables (for instance, attack is characterised by distance and speed of the bot to characters in the centre of his field of view), and triggering recognition at several timescales.

Behaviours are recognised after a delay (backpropagating a state to past, yet unrecognised events, thanks to a critical event like picking a health bonus), after examining critical variables over a fixed period (to identify danger checking behaviours, for instance), or immediately on special events (like attacking and fleeing). Exploration is a default state, when no other seems to match the observations.

We do this recognition off-line, on data representing ten to fifteen minutes of game-play; processing this data and producing the tables that represent our behaviour takes five to ten seconds.

\subsection{Results}

\begin{tabular}{|c|c|c|}
\hline recognition learned, aggressive & 4.4 \\
\hline recognition learned, cautious & 13.9 \\
\hline selection learned, aggressive & 45.7 \\
\hline manual specification, aggressive & 8.0 \\
\hline manual specification, cautious & 12.2 \\
& manual specification, uniform & 43.2 \\
\hline Table 3 & native (level 3/8) UT bot & 11.0 \\
\hline
\end{tabular}

Performance comparison on learned, hand-specified, and native Unreal bots (lower is better)

Table 3 shows a comparison between different specification methods. Numbers are the average difference to the winning bot, over ten games won by the first bot reaching 100 kills. Our bots compare well to the native Unreal Tournament bot, whose skill corresponds to an average human player. Aggressive bots (gray lines) perform significantly better, and learning by recognition does much better than learning by selection, along with hand specification.

Lessons from these results can be summed up in the following way (we will refer here to table 4, which is the same as table 1 , but learnt by recognition):

(1) learnt tables share common aspects with hand-written tables (as for the transition table $\left.P\left(S_{t+1} \mid S_{t}\right)\right)$; for instance,in the fleeing state $F$, health level is much more probably low or medium than high;

(2) differences in behaviour of the teacher influence the learnt behaviour: aggressivity (or the lack of it) is found in the learnt behaviour, and translates into performance variations (in our setup, aggressive behaviours seem to be more successful);

(3) nevertheless, differences between handspecified and learnt models are noticeable; they can explained by:

(a) player-specific behaviours: humans almost always attack and do not retreat; another example is the low probability of $P\left(H=H i g h \mid S_{t+1}=S W\right)$ in the learnt table (dark gray cell on table 4$)$ : it can be explained by the fact that human players give a much higher priority to searching a good weapon over searching for health bonuses;

(b) additional information: some parts of the hand-written tables are specified as uniform (as a result from a refusal or impossibility to specify theoretically a link between two events, like the value of the opponent's weapon knowing that the bot is exploring), whereas their learnt counterparts include information;

(c) perceptive differences: a human player and a bot have a different perception of sound (the human perceives direction combined with the origin of sound, like an impact on a wall or the sound of the shooting itself, whereas the bot senses only direction);

(d) bias induced by data quantity: a human player has almost always a medium 
health level (which is due to a poor choice of discretisation for the health level variable), which explains higher values in the learnt table 4 (line of light gray cells);

(4) our learning methods lead to functioning behaviours; learning using behaviour recognition scores best, and allows to reach the level of an average native UT bot.

\section{Discussion}

\subsection{Evaluation}

We shall now come back to the objectives we listed at the beginning, to try and assess our method in practical terms.

\subsubsection{Development Team's Viewpoint}

Programming efficiency. Our method of behaviour design relies upon a clear theoretical ground. Programming the basic model can use a generic bayesian programming library, and needs afterwards little more than the translation into $\mathrm{C}++$ (for instance) of the mathematical model. Design is really expressed in terms of practical questions to the expertise of the designer, like "if the bot is attacking, how high is his health level?"; it does not require a preliminary formalisation of the expected behaviour to program. Moreover, in our model behaviours are data (our tables). It means that they can easily be loaded and saved while the behaviour is running, or exchanged amongst a community of players or developers.

Limited computation requirements. The computation time needed for a decision under our model can be shown to be linear in both the number of sensory variables and the number of states.

Design / Development separation. Development amounts to incorporating the bayesian framework into the control architecture, and establishing the bayesian model; design consists in establishing relations between the variables in the form of probability distributions. A designer really has to know about what the bot should do, but does not need any knowledge of the implementation details; he needs but a light background on probabilities, and no scripting or programming at all.
Behaviour tunability. We have seen that our way of specifying behaviours gives a natural way to formalise human expertise about behaviours, and that it implies that tuning a behaviour is possible, as they are expressed in natural terms and not in artificial logical or scripting terms. Moreover, the quantity of data needed to describe a behaviour is kept small compared to an FSM, and this helps keeping the analysis and control of a behaviour tractable for the designer.

\subsubsection{Player's Viewpoint}

"Humanness". This criterion is hard to assess, although it can be done [13] in ways comparable to the Turing test [14]. Our method of specifying a behaviour helps the designer translate his expertise easily, and therefore gives him a chance to build a believable bot.

Behaviour learning. We have seen that learning under our model is natural: it amounts to measuring frequencies. This is a chance for the player to teach its teammate bots how to play. Recognising high-level states on the basis of low-level commands is possible, and allows a player to adjust a behaviour completely transparently, with the original controls of the game.

\subsection{Perspectives}

We have shown a way to specify FSM-like action selection models for virtual robots, and to learn these models by example. The recognition involved in learning from the natural actions of a player in the game remains a classicaly programmed heuristic; an obvious perspective is to formalise it within the bayesian framework, in order to perform probabilistic behaviour recognition. This would grant more adaptability to variations in the behaviour model.

\section{Acknowledgments}

This work was supported by the ROBEA-CNRS project "Modeles Bayesiens pour la Generation de Mouvement", the BIBA project funded by the European Community, and a grant from the French Ministry of Research. 
Table 4

\begin{tabular}{|c|r||r||r||r||r||r|}
\hline$H \backslash S_{t+1}$ & $A$ & $S W$ & $S H$ & $E x$ & $F$ & $D D$ \\
\hline Low & 0.179 & 0.342 & 0.307 & 0.191 & 0.457 & 0.033 \\
\hline Medium & 0.478 & 0.647 & 0.508 & 0.486 & 0.395 & 0.933 \\
\hline High & 0.343 & 0.011 & 0.185 & 0.323 & 0.148 & 0.033 \\
\hline
\end{tabular}

learnt $P\left(H \mid S_{t+1}\right)$

\section{References}

[1] J. E. Laird, M. Van Lent, Human-level AI's killer application : Interactive computer games, in: AAAI Fall Symposium Technical Report, 2000.

[2] G. A. Kaminka, M. Veloso, S. Schaffer, C. Sollitto, R. Adobbati, A. N. Marshal, S. Scholer, Andrew, S. Tejada, Gamebots: the ever-challenging multiagent research test-bed, Communications of the ACM.

[3] J. E. Laird, It knows what you're going to do : Adding anticipation to a quakebot, in: AAAI Spring Symposium Technical Report, 2000.

[4] S. Woodcock, Game AI : The state of the industry 2000-2001, Game Developer.

[5] J. Laird, Design goals for autonomous synthetic characters, draft (2000).

[6] O. Lebeltel, P. Bessière, J. Diard, E. Mazer, Bayesian Robot Programming, Autonomous RobotsIn press.

[7] E. T. Jaynes, Probability theory: the logic of science, unprinted book, available on-line at http://bayes.wustl.edu/ (1995).

[8] M. Jordan (Ed.), Learning in Graphical Models, MIT Press, 1998.

[9] G. Cooper, The computational complexity of probabilistic inference using bayesian belief network, Artificial Intelligence 42 (2-3).

[10] E. Dysband, A finite-state machine class, in: M. Deloura (Ed.), Game Programming Gems, Charles River Media, 2000, pp. 237-248.

[11] M. Zarozinski, Imploding combinatorial explosion in a fuzzy system, in: M. Deloura (Ed.), Game Programming Gems 2, Charles River Media, 2001, pp. $342-350$.

[12] Unrealscript language reference, Website,

http://unreal .epicgames . com/UnrealScript .htm.

[13] J. E. Laird, J. C. Duchi, Creating human-like synthetic characters with multiple skill-levels : A case study using the Soar quakebot, in: AAAI Fall Symposium Technical Report, 2000.

[14] A. M. Turing, Computing machinery and intelligence, Mind 59 (236) (1950) 433-460. 